\title{
Review of: "Feasibility of Using Wearable EMG Armbands combined with Unsupervised Transfer Learning for Seamless Myoelectric Control"
}

\author{
helene Geng ${ }^{1}$ \\ 1 Shenzhen Institutes of Advanced Technology - Chinese Academy of Sciences
}

Potential competing interests: The author(s) declared that no potential competing interests exist.

Aiming at providing robust yet seamless myoelectric control, this paper proposed a SCADANN algorithm to examine the motion classification performance across multi-user, multi-days, and multi-location based on Myo sensor. The manuscript is well motivated and written. The following are some concerns of the reviewer about the methods and results.

\# 1: This paper used deep neural network with TSD, and found that it worked better than Convnet. However, the authors did not discuss in detail why it worked better than ConvNet, so further discussion and explanation should be made. In addition, according to the description in Figure 2, the neural network model with TSD consists of three fully connected layers. A neural network with only three layers cannot be considered a "deep" neural network. If it is not clearly expressed in Figure 2, the authors should further explain the neural network model, or correct the statement.

\# 2: In Figure 4, the authors showed a series of experiments. However, it is suggested that the authors should supplement the following experiments: simultaneously satisfy two or three of the three conditions (multi-users, multi-days, multi-location). For example, in the case of both multi-users and multi-days, test the performance of the algorithm.

\# 3: In Figure 4, the authors showed the performance of the algorithm. However, specific data and numerical values lacked. The authors should mark specific values in Figure 4 (e.g. 78\%), or additional display a data table to quantify results.

\# 4: The authors should add Section 2.4 to explain the evaluation criteria and evaluation parameters for the experiments in this work, e.g. accuracy and correlation. 